\title{
Isolation by using albumin columns of a cohort of fast-swimming human spermatozoa*
}

\author{
Ø. Magnus $\dagger$, A. Tolefsrud $\ddagger$, T. Åbyholm $\dagger$ and K. Purvis $\ddagger$ \\ Department of $\dagger$ Obstetrics and Gynecology and $\ddagger$ Institute of Pathology, National Hospital, \\ University of Oslo, Oslo, Norway
}

\begin{abstract}
Summary. Unwashed human spermatozoa enter columns of $20 \%$ albumin in cohorts and not in a continuous stream. Using normospermic ejaculates, a single cohort, representing $\sim 10 \%$ of the total number of spermatozoa added, appeared within $1 \mathrm{~h}$ of addition. In contrast, when ejaculates from asthenozoospermic men were tested using the same incubation period $(1 \mathrm{~h})$, the cohorts contained $<5 \%$ of the total. Exposure of ejaculates to 2-deoxyadenosine, a potent stimulator of sperm motility, doubled the number of spermatozoa present in the cohort, but not the average velocity of the group. We suggest that spermatozoa also enter the cervical mucus and pass through the female reproductive tract in cohesive groups.
\end{abstract}

Keywords: spermatozoa; albumin columns; cohorts; 2-deoxyadenosine; asthenozoospermia; man

\section{Introduction}

Several groups have observed that, after spermatozoa have penetrated the cervical secretion, they have a tendency to enter the uterine cavity as cohesive groups rather than in a continuous stream (Settlage et al., 1973; Elstein, 1978; Odeblad, 1978). Albumin columns have long been used to isolate spermatozoa with a superior capacity for progressive movement (Urry et al., 1983). During our own attempts to enrich sperm samples using these columns, we noticed that the pattern of spermatozoa in the column appeared to follow a normal distribution, instead of a linear gradient decreasing from top to bottom. This finding implied that a cohort of fast-swimming cells had broken away from the main group and entered the protein solution, thus lending experimental support to the observations in vivo. The data presented in this article represent preliminary studies on this cohort.

\section{Materials and Methods}

The semen samples were, in the majority of cases, provided by medical students of proven fertility. In one study, ejaculates from a random group of normal (sperm count $>40 \times 10^{6} / \mathrm{ml}$, motility $>40 \%$, grade 2 ) and asthenozoospermic men (normal count and motility $<40 \%$, grade 2 ) attending our infertility clinic were tested.

Preparation and use of albumin columns. A disposable plastic syringe ( $1 \mathrm{ml}$ ) was filled with albumin solution. In the majority of cases, the columns contained a $20 \%$ bovine serum albumin (BSA) solution ( $4 \mathrm{~g} / 20 \mathrm{ml}$ Earles balanced salt solution) neutralized to $\mathrm{pH} \mathrm{7.2-7.5}$ with $1 \mathrm{~N}-\mathrm{NaOH}$. In one study, solutions of $5 \%, 10 \%$ and $15 \%$ BSA were also prepared. An aliquant $(200 \mu \mathrm{l})$ of freshly liquefied semen was then carefully applied to the top of the column, which was held in a vertical position for $1 \mathrm{~h}$ (in one study additionally $\mathrm{l} .5$ and $2 \mathrm{~h}$ ) with the column contents stationary. After incubation, the column contents were divided into 10 , or in one study 15 , fractions, equivalent to $10 \mathrm{drops}(100 \mu \mathrm{l})$ or 7

\footnotetext{
* Reprint requests to Dr K. Purvis.
} 
drops $(70 \mu \mathrm{l})$ respectively, by simply allowing the solution to drip into a series of glass tubes. The fractions were then stored at $-20^{\circ} \mathrm{C}$ and later counted using a Bürker chamber.

Cohort studies. The effects of varying the albumin concentration of the column medium on the passage of living and dead spermatozoa were tested using the same incubation time of $1 \mathrm{~h}$. Columns were prepared with $5 \%$, $10 \%, 15 \%$ and $20 \%$ BSA solution and aliquants of the same normal ejaculate were run in parallel. Additional aliquants were frozen at $-20^{\circ} \mathrm{C}$ for $10 \mathrm{~min}$ and run as dead controls. After fractionation, the total numbers of spermatozoa present in the individual cohorts were computed and expressed as a percentage of the number of cells introduced to the top of each column. In some cases, aliquants were also mixed directly with the different BSA solutions and incubated for $1 \mathrm{~h}$ to determine the direct effects on sperm motility. Sperm movement was assessed using a sperm motility analyser (Lazymot, BTG, Dusseldorf, F.R.G.), a technique based on laser-Doppler analysis (Pusch, 1985).

To evaluate the effects of incubation time on the size and movement pattern of the cohort, aliquants of the same normozoospermic ejaculate were run on $20 \%$ BSA columns for $1,1.5$ and $2 \mathrm{~h}$. The column eluates were then divided into 15 fractions so that small differences in the sperm distribution could be discerned.

Semen samples from 3 donors of proven fertility and 3 asthenozoospermic men were run for $1 \mathrm{~h}$ on columns containing $20 \%$ BSA. To facilitate a better comparison similar numbers of spermatozoa $\left(5 \times 10^{6}\right.$ cells $)$ were added to the columns.

Samples of normozoospermic ejaculates $(100 \mu \mathrm{l})$ were mixed in equal proportions with $5 \mathrm{~mm}$-2-deoxyadenosine (Sigma Chemical Co., St Louis, MO, U.S.A.), an acknowledged stimulator of sperm movement (Aitken et al., 1986), in Earles medium, incubated for $30 \mathrm{~min}$ and then added to albumin columns containing $20 \%$ BSA and $2.5 \mathrm{~mm}$-2-deoxyadenosine. Control runs were carried out using semen aliquants diluted $1: 1$ (v/v) with medium on columns containing only BSA. Each pair was incubated for the same time $(1 \mathrm{~h})$ and divided into 10 fractions.

\section{Results}

In the case of the 5 and $10 \%$ BSA columns, the viscosity of the medium was not sufficient to support the semen aliquant which became gradually mixed with the column material. At BSA concentrations of $15 \%$, spermatozoa moved relatively unhindered through the column with a tendency to form more than one cohort (Fig. 1). Increasing the protein concentration further to $20 \%$ with an incubation period of $1 \mathrm{~h}$ encouraged the spermatozoa to form a single cohort which eluted in the middle of the column. Under these conditions, the cohort was sufficiently separated from the main group to enable an extrapolation of its profile to the $x$ axis. This facilitated calculation of its size relative to the total number of spermatozoa introduced to the column.

Figure 2 shows the distribution of live and dead (cold-shocked) spermatozoa in a $20 \%$ BSA column after $1 \mathrm{~h}$ incubation. As indicated, dead cells entered the column in small numbers, decreasing exponentially from top to bottom. Those that penetrated the column were invariably in the form of large aggregates. In contrast, living spermatozoa entered in relatively large numbers and followed a bell-shaped distribution distinctly separated from the main body of spermatozoa. In this study, using semen from a normospermic subject, the cohort constituted about $10 \%$ of the total number of spermatozoa added.

Incubation of spermatozoa in the various protein solutions caused a reduction in progressive velocity with increasing albumin concentration. The number of motile spermatozoa and their velocity in $20 \%$ BSA was approximately $50 \%$ of that in medium alone (Table 1 ).

Increasing the incubation time from 1 to $2 \mathrm{~h}$ was associated with the appearance of a new cohort of cells which gradually separated from the primary cohort (Fig. 3). There was no evidence for the passage of new cohorts of cells from the semen aliquant into the protein solution. An incubation time of $1 \mathrm{~h}$ was chosen for future studies since it gave the best visualization of the sperm cohort. The penetration of the albumin columns by spermatozoa from normozoospermic and asthenozoospermic men occurred to different degrees. The number of spermatozoa present in the cohort from the normozoospermic subjects represented $7.6 \%, 10.9 \%$ and $13.9 \%$ of the total added. The corresponding values for the asthenozoospermic men were $3.9 \%, 4 \cdot 6 \%$ and $4.8 \%$. Examples of representative distributions are shown in Fig. 4. The average velocities for both groups were similar, as indicated by the relative positions of the cohorts on the columns. 


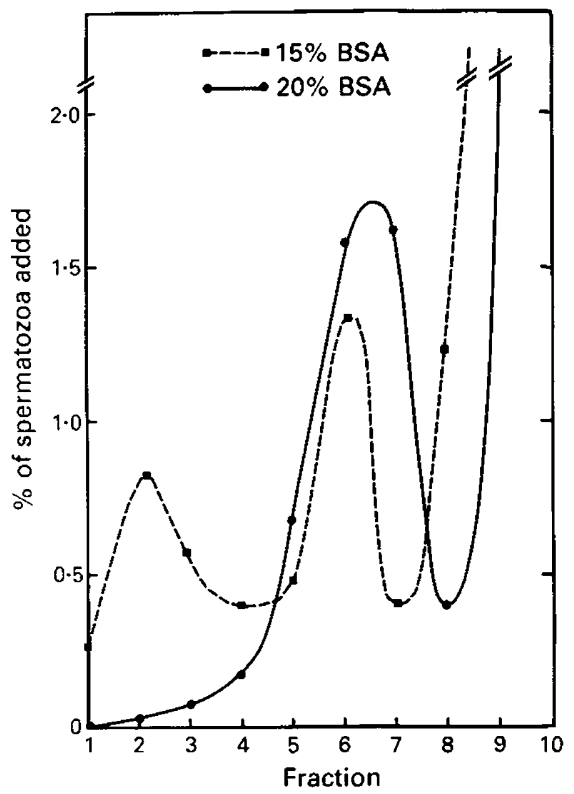

Fig. 1

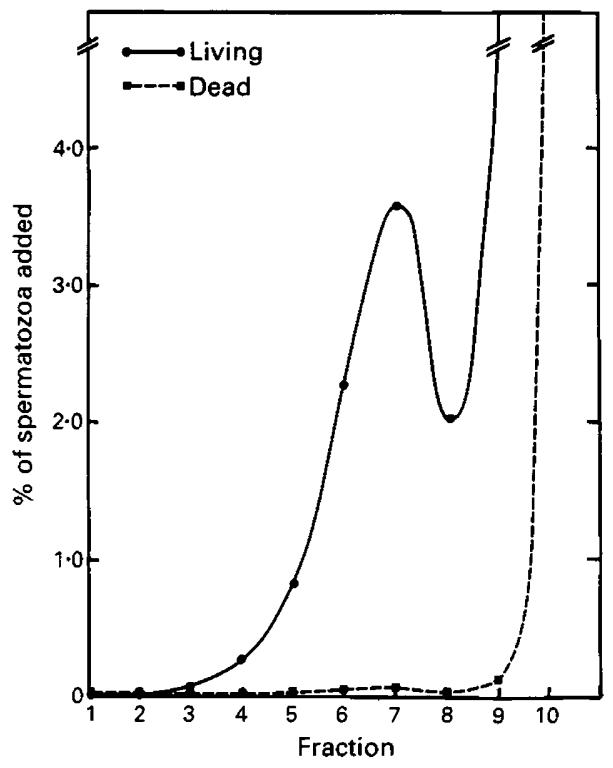

Fig. 2

Fig. 1. Distribution of spermatozoa in columns $(1 \mathrm{ml})$ of Earles medium containing $15 \%$ or $20 \%$ bovine serum albumin after $1-\mathrm{h}$ incubation at room temperature. Aliquants $(200 \mu \mathrm{l})$ of semen from a normozoospermic donor were applied to the column and its contents were collected in 10 fractions.

Fig. 2. Distribution of spermatozoa in an albumin column $(1 \mathrm{ml})$ after $1-\mathrm{h}$ incubation at room temperature. Aliquants $(200 \mu \mathrm{l})$ of the same ejaculate, freshly liquefied $(-0)$ or cold-treated $(\square---\mathbf{E})$, were applied to the column and its contents were later divided into 10 fractions.

Table 1. Sperm motility characteristics from 3 patients $(A, B, C)$ after incubation in control medium (C), $10 \%$ BSA and $20 \%$ BSA

\begin{tabular}{|c|c|c|c|c|c|c|c|c|c|c|c|c|}
\hline \multirow[b]{2}{*}{ Patient } & \multicolumn{3}{|c|}{$\begin{array}{l}\% \text { motile } \\
\text { cells }\end{array}$} & \multicolumn{3}{|c|}{$\begin{array}{l}\text { Velocity of } \\
\text { motile cells } \\
(\mu \mathrm{m} / \mathrm{sec})\end{array}$} & \multicolumn{3}{|c|}{$\begin{array}{l}\% \text { Progressive } \\
\text { mobility }\end{array}$} & \multicolumn{3}{|c|}{$\begin{array}{c}\text { Velocity of } \\
\text { progressively } \\
\text { mobile cells } \\
(\mu \mathrm{m} / \mathrm{sec})\end{array}$} \\
\hline & $\mathrm{C}$ & 10 & 20 & C & 10 & 20 & C & 10 & 20 & C & 10 & 20 \\
\hline A & 66 & 56 & 49 & 35 & 26 & 23 & 25 & 14 & 8 & 63 & 52 & 49 \\
\hline B & 57 & 48 & 23 & 36 & 29 & 25 & 25 & 16 & 10 & 65 & 55 & 51 \\
\hline $\mathrm{C}$ & 86 & 53 & 35 & 44 & 31 & 21 & 45 & 22 & 6 & 71 & 60 & 50 \\
\hline
\end{tabular}

Figure 5 shows a typical column distribution of spermatozoa from normozoospermic men after exposure to 2-deoxyadenosine. The number of cells in the cohort was approximately doubled, but its relative position indicated that the drug did not alter the average velocity of the group. Using semen from 4 subjects, the proportions of spermatozoa present in the cohort were $6 \cdot 3 \%, 7 \cdot 2 \%$, $8.2 \%$ and $8.3 \%$ in the absence of the compound. The corresponding values for aliquants of the same semen samples incubated with 2-deoxyadenosine were $12 \cdot 4 \%, 13 \cdot 3 \%, 15.7 \%$ and $16.9 \%$, respectively. 


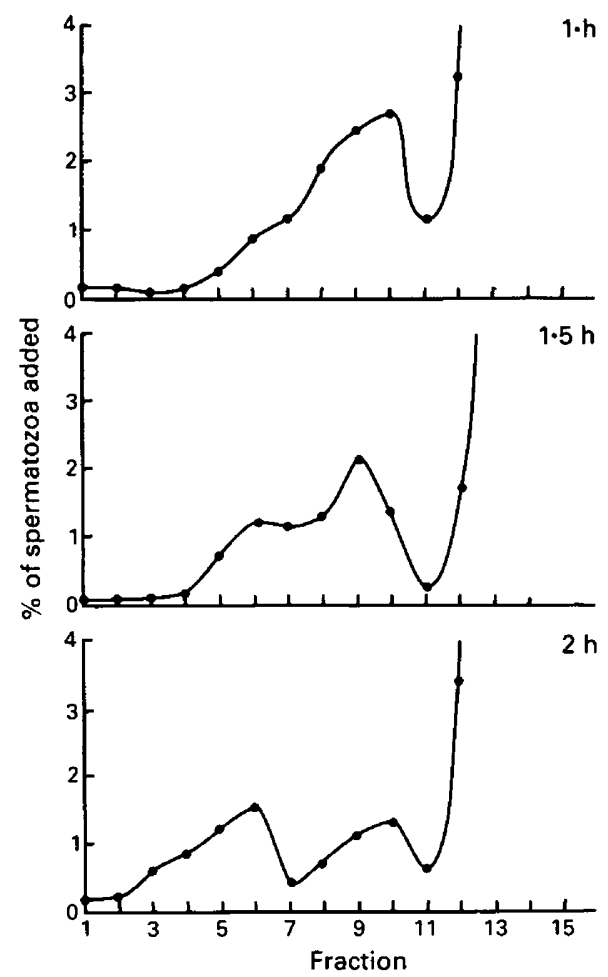

Fig. 3. Effects of incubation time $(1 \mathrm{~h}, 1.5 \mathrm{~h}$ and $2 \mathrm{~h})$ on the distribution of spermatozoa from the same normozoospermic donor in an albumin column $(1 \mathrm{ml})$. In this case, the eluate was divided into 15 fractions.

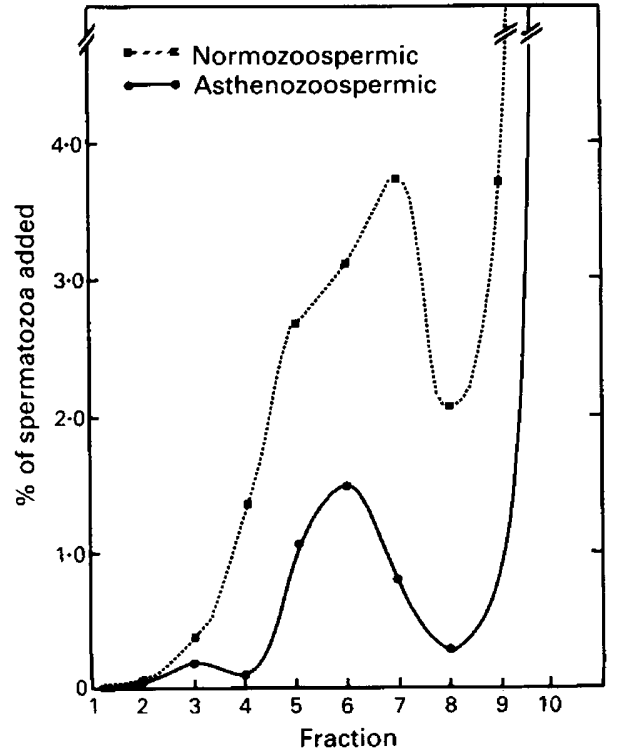

Fig. 4

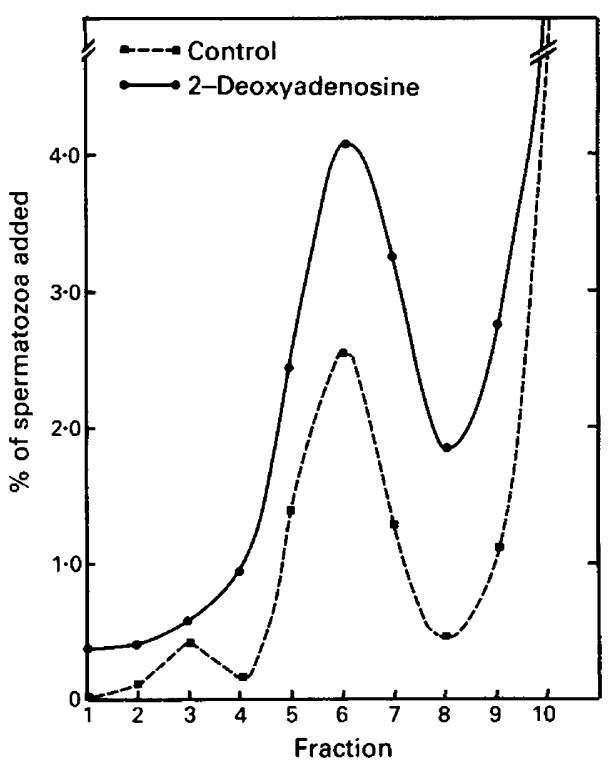

Fig. 5

Fig. 4. Distribution of spermatozoa from normozoospermic and asthenozoospermic individuals in albumin columns $(1 \mathrm{ml})$ after 1 -h incubation at room temperature.

Fig. 5. Effects of incubation with and without $2.5 \mathrm{~mm}$-2-deoxyadenosine on the distribution of spermatozoa in albumin columns. Ejaculates were provided by fertile donors. 


\section{Discussion}

Various methods have been used to isolate spermatozoa with a superior swimming ability from ejaculates of poor quality, for purposes of insemination (Glass \& Ericsson, 1978; Allen et al., 1985; Jeyendren et al., 1986; Russell \& Rogers, 1987). One of the techniques involves the application of a sperm sample onto columns of relatively viscous protein solutions, often albumin, which serve to hinder the passage of abnormal or poorly motile cells (Urry et al., 1983). In the majority of cases, the spermatozoa are first washed free from the seminal plasma using centrifugation. To avoid the potentially adverse effects of these washing procedures on sperm quality, our own attempts at isolating superior swimmers on albumin columns used spermatozoa which had not been subjected to any prior manipulation. Under these conditions, it became apparent that those spermatozoa entering the protein matrix did so as a cohesive group and did not follow a continuous gradient from the top to the bottom of the column. On the other hand, spermatozoa killed by cold shock did not enter the albumin in significant quantities. Subsequent studies revealed that an albumin concentration of $20 \%$ was optimal for visualization of the cohesive group of penetrating sperm cells. When spermatozoa were incubated in medium containing $20 \%$ albumin for $1 \mathrm{~h}$, the percentage of motile spermatozoa and their average velocity was reduced by more than half, suggesting that the high viscosity of the medium suppresses the movement of spermatozoa with a low motility potential. Increasing the incubation time or reducing the protein concentration of the medium was associated with the formation of a new cohort which broke away from the primary group. The reasons for this are unclear. It may indicate that some of the spermatozoa have a limited capacity for movement in the viscous medium and are soon exhausted by the effort. According to Lee et al. (1977), leading swimmers may pave the way for other spermatozoa by reducing the friction in the medium. Those following then relieve the leaders when they become exhausted.

The ejaculates of asthenozoospermic men contained a lower percentage of spermatozoa capable of overcoming the resistance offered by the seminal plasma-albumin interface. Once entering the protein solution these cells followed the same distribution as spermatozoa from normozoospermic men, thus swimming with the same velocity. Such observations indicate that the use of techniques to enrich the ejaculates of asthenozoospermic men, before insemination, may prove to be fruitful. Indeed, clinical experience suggests that, in spite of this generally poor sperm motility, the pure asthenozoospermic patient has a better prognosis for fertilization after insemination with washed spermatozoa than do those with oligo-astheno-teratozoospermia (Belker \& Cook, 1987).

It was possible to recruit more cells in the cohort by incubation with 2-deoxyadenosine, one of the most potent stimulators of sperm motility (Aitken et al., 1986). This effect appears to be mediated through the sperm adenylate cyclase, by virtue of its effects on intracellular cAMP levels (Vijayaraghavan \& Hoskins, 1986). However, the average velocity of the whole group remained unchanged, suggesting that, under normal conditions, the spermatozoa present in the cohort are already maximally stimulated. Indeed, this may represent a precondition for the cells to cross the seminal plasma-albumin interphase.

Bedford (1975) has noted that "The release of spermatozoa from the cervical pool occurs sequentially over a prolonged period". Measurements of the distribution of spermatozoa throughout the female reproductive tract a short time after ejaculation reveal a sequential build up of cells in successive regions (El-Banna \& Hafez, 1970; Overstreet et al., 1978). Although it has been argued that this probably is related to the specialized structure of the cervical mucus (Odeblad, 1978; Moghissi, 1984), the possibility that it may also reflect an inherent property of the germ cells has also been raised (Mortimer, 1978). The results of the present studies, which indicate that human spermatozoa swim in cohorts in viscous protein media, lend support to the latter possibility. Although the use of albumin columns may be considered unphysiological, they may provide a simple model for studying a potentially natural phenomenon. 
We thank the Norwegian Research Council for Science and the Humanities (NAVF) for financial support of these studies.

\section{References}

Aitken, R.J., Mattei, A. \& Irvine, S. (1986) Paradoxical stimulation of human sperm motility by 2-deoxyadenosine. J. Reprod. Fert. 78, 515-527.

Allen, N.C., Herbert, C.M., Maxson, W.S., Rogers, B.J., Diamond, M.P. \& Wentz, A.C. (1985) Intrauterine insemination. A critical review. Fert. Steril. 44, 569-580.

Bedford, J.M. (1975) Sperm transport, capacitation and fertilization. In The Male Reproductive System, Handbook of Physiology. Endocrinology; Volume V, pp. 338-392. Eds D. W. Hamilton \& R. O. Greep. American Physiological Society, Washington D.C.

Belker, A.M. \& Cook, C.L. (1987) Sperm processing and intrauterine insemination for oligospermia. Urol. Clin. North Am. 14, 597-607.

El-Banna, A.A. \& Hafez, E.S.E. (1970) Sperm transport and distribution in rabbit and cattle female tract. Fert. Steril. 21, 534-540.

Elstein, M. (1978) Functions and physical properties of mucus in the female genital tract. Br. med. Bull. 34, $83-88$.

Glass, R.H. \& Ericsson, R.J. (1978) Intrauterine insemination of isolated motile sperm. Fert. Steril. 29, 535-538.

Jeyendran, R.S., Perez-Pelaez, M. \& Crabo, B.G. (1986) Concentration of viable spermatozoa for artificial insemination. Fert. Steril. 45, 132-134.

Lee, W., Blandau, R.J. \& Verdugo, P. (1977) Laser lightscattering studies of cervical mucus. In The Uterine Cervix in Reproduction, pp. 68-82. Eds V. Insler \& G. Bettendorf. Georg Thieme, Stuttgart.

Moghissi, K.S. (1984) The function of the cervix in human reproduction. Year Book Medical Publishers, 1984, 4-58.
Mortimer, D. (1978) Selectivity of sperm transport in the female genital tract. In Spermatozoa, Antibodies and Infertility, pp. 37-53. Eds J. Cohen \& W. F. Hendry. Blackwell, Oxford.

Odeblad, E. (1978) Cervical factors. Contr. Gynec. Obstet. 4, 132-142.

Overstreet, J.W., Cooper, G.W. \& Katz D.F. (1978) Sperm transport in the reproductive tract of the female rabbit: II. The sustained phase of transport. Biol. Reprod. 19, 115-132.

Pusch, H.H. (1985) Messung der Spermatozoen motilität mittels Laser-Doppler-Spektroskie im andrologischen Routine-labor. Andrologia 17, 217-223.

Russell, L.D. \& Rogers, B.J. (1987) Improvement in the quality and fertilization potential of a human sperm population using the rise technique. J. Androl. 8, 25-33.

Settlage, D.S., Motoshimo, M. \& Tredway, D.R. (1973) Sperm transport from the external cervix os to the fallopian tubes in women. A time and quantitation study. Fert. Steril. 24, 655-661.

Urry, R.L., Middleton, R.G., McNamara, L. \& Vikari, C.A. (1983) The effect of single-density bovine serum albumin columns on sperm concentration, motility, and morphology. Fert. Steril. 40, 666-669.

Vijayaraghavan, S. \& Hoskins, D.D. (1986) Regulation of bovine sperm motility and cyclic adenosine $3^{\prime}, 5^{\prime}$. monophosphate by adenosine and its analogues. Biol. Reprod. 34, 468-477. 